\title{
Absolute Stereostructures by Quantum Chemical Electronic Circular Dichroism Calculations of Unusual Natural Products
}

\author{
Moses K. Langat
}

Natural Products Research Group, Department of Chemistry, University of Surrey, Guildford, Surrey, GU2 7XH, UK

South African Croton gratissimus Burch. var. gratissimus (Euphorbiaceae), Russian Pinus pumila (Pall.) Regel (Pinaceae) and Madagascan Tachiadenus longiflorus Griseb. (Gentianaceae) gave unusual natural products, gratissimol (1), [1] pumilol (2) and langaside (3) respectively whose skeleta have not been reported previously. The absolute configurations of $\mathbf{1}, \mathbf{2}$ and $\mathbf{3}$ were determined by comparing their theoretically calculated and experimental ECD curves. Conformational searches were done on possible isomers that were consistent to correlations in their NOESY spectra using MMFF basis set built into Spartan08 software and conformers which were under $2 \mathrm{kcal} / \mathrm{mol}$ subjected to TDDFT calculations using a B3LYP method at 6-31G (d, f) level built into Gaussian09 software. The resulting ECD curves of the selected conformers were Boltzmann weighted and compared to the experimental ECD curves for 1, 2 and $\mathbf{3}$.
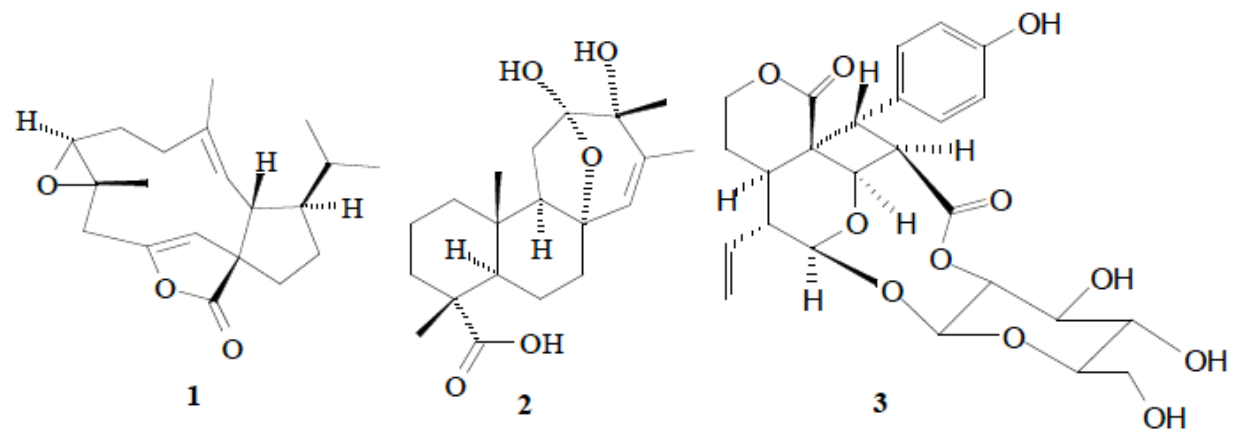

\section{REFERENCES}

